\title{
Lack of association between COX-2 staining level and biochemical recurrence following salvage radiation therapy for recurrent prostate cancer
}

\author{
Michael G. Heckman • Katherine S. Tzou • Alexander S. Parker • \\ Thomas M. Pisansky • Steven E. Schild • Tracy W. Hilton • Vivek N. Patel • \\ Liset Pelaez • Li Yan Khor • Jennifer L. Peterson • Larry C. Daugherty • \\ Laura A. Vallow • Alan Pollack • Steven J. Buskirk
}

Received: 31 October 2012 / Accepted: 20 March 2013 /Published online: 3 April 2013

(C) Springer-Verlag Berlin Heidelberg 2013

\begin{abstract}
Objective The ability to predict which men will experience biochemical recurrence (BCR) after salvage radiation therapy (SRT) for recurrent prostate cancer following radical prostatectomy has potential for improvement. Cyclooxygenase- 2 (COX-2) overexpression has previously correlated with poor clinical outcomes following primary treatment for prostate cancer; however, its predictive ability in the specific setting of SRT has not been examined to date. This study evaluated the association between COX-2 staining intensity and BCR following SRT for recurrent prostate cancer.

Methods We utilized a cohort of 151 patients who underwent SRT between July 1987 and July 2003. COX-2 staining intensity in primary tumor samples was detected using monoclonal antibodies and quantified using a computer-assisted
\end{abstract}

Electronic supplementary material The online version of this article (doi:10.1007/s13566-013-0099-2) contains supplementary material, which is available to authorized users.

\footnotetext{
M. G. Heckman ( $\square)$

Section of Biostatistics, Mayo Clinic Florida, 4500 San Pablo

Road, Jacksonville, FL 32224, USA

e-mail: heckman.michael@mayo.edu

K. S. Tzou • J. L. Peterson • L. C. Daugherty $\cdot$ L. A. Vallow

S. J. Buskirk

Department of Radiation Oncology, Mayo Clinic Florida,

Jacksonville, FL, USA

A. S. Parker · T. W. Hilton

Department of Epidemiology, Mayo Clinic Florida, Jacksonville, FL, USA

T. M. Pisansky

Department of Radiation Oncology, Mayo Clinic Rochester,

Rochester, MN, USA
}

method. The association between COX-2 staining intensity and BCR was evaluated using multivariable Cox regression models.

Results When examining COX-2 staining level as threelevel categorical variable (low, moderate, high) based on approximate sample tertiles, there was no evidence of an association with BCR $(P=0.18)$. More specifically, in comparison to patients with low staining intensity, there was no significant difference in risk of BCR for moderate (relative risk [RR], $1.17 ; P=0.56)$ or high $(\mathrm{RR}, 0.72 ; P=0.22) \mathrm{COX}-2$ staining intensity patients. This lack of association was also observed when considering COX-2 staining intensity as a continuous variable (RR, $0.83 ; P=0.15)$.

Conclusion Our results indicate that COX-2 staining intensity is likely of little use in discriminating prognosis of SRT.

\author{
S. E. Schild \\ Department of Radiation Oncology, Mayo Clinic Arizona, \\ Scottsdale, AZ, USA \\ V. N. Patel \\ Miller School of Medicine, University of Miami, Miami, FL, USA \\ L. Pelaez \\ Department of Pathology, University of Miami, Miami, FL, USA \\ L. Y. Khor \\ Anatomic Pathology, Cleveland Clinic Foundation, Cleveland, \\ $\mathrm{OH}$, USA \\ A. Pollack \\ Radiation Oncology, University of Miami, Miami, FL, USA
}


It appears that the search for prognostic factors associated with BCR should continue elsewhere in order to further enhance patient selection for SRT.

Keywords Prostate cancer - Salvage radiation therapy · Biochemical recurrence $\cdot$ Tumor biomarker

\section{Introduction}

In the USA, approximately 15,000 men who have undergone radical prostatectomy for localized prostate cancer will experience a significant rise in their serum prostate-specific antigen (PSA) level each year [1], indicating biochemical recurrence (BCR). Salvage radiation therapy (SRT) is the only potentially curative treatment for these men, and without it more than $35 \%$ will develop distant metastases within 5 years [2], while between 25 and $40 \%$ will die due to prostate cancer within 10 years [3, 4]. Reported SRT success rates range from 10 to $50 \%$ [5-9], and therefore enhancements of the ability to select men who are most likely to respond to treatment are of great importance. BCR is the first sign of disease progression following SRT and in the absence of hormone therapy is followed by development of distant metastases at an average of 8 years later [2]. Clinicopathologic parameters have been evaluated for association with BCR following SRT by a number of groups, with Gleason score, pathological tumor stage, pre-SRT PSA, and other factors showing evidence of association [5]. Additionally, several groups, including our own, have created scoring algorithms that combine information from these key clinical and pathological features in order to assign patients different levels of risk of BCR [6, 7].

Though the results of the aforementioned investigations can be very helpful in the identification of patients most likely to respond to SRT, the continued evaluation of plausible prognostic factors for BCR is important in order to further improve patient selection. In addition to their potential utility in this capacity, the study of tumor-based biomarkers in relation to SRT outcome can aid in the search for adjuvant therapies to be used in combination with SRT and also add to our understanding of prostate cancer progression. Our group has evaluated a number of tumor-based biomarkers in regard to association with BCR following SRT; both Ki-67 and B7-H3 have shown significant associations with recurrence, whereas no predictive ability was evident for MDM2, p16, or p53 [8-10].

The cyclooxygenase-2 (COX-2) enzyme is another promising candidate for study in relation to response to SRT, especially due to its potential as a target for adjuvant therapy. Well-known for its role in inflammation, studies have shown that COX-2 is also over-expressed in several human cancers, including prostate cancer, and appears to play an important role in prostate cancer development and progression [11]. Several investigations have demonstrated associations between elevated COX-2 expression and poor prostate cancer clinical outcomes [12-16]. However, no study to date has examined the ability of COX-2 expression to predict BCR in men undergoing SRT due to recurrent prostate cancer following radical prostatectomy. Therefore, the aim of this study was to evaluate the association between COX-2 staining level in primary prostate cancer tumors and BCR following SRT.

\section{Methods}

Patient selection and outcome definition

This study was approved by the Mayo Clinic Institutional Review Board. The same underlying cohort of 368 patients that was used to develop our previously referred to scoring algorithm (the BCR Risk Score) [6] was utilized in the current study. These were all men who underwent SRT to treat a rising PSA following surgery for prostate cancer at the Mayo Clinic between July 1987 and July 2003. A total of $151(41 \%)$ men from this original cohort had archived tumor tissue available and were therefore included in the current study. We observed no dramatic differences when comparing clinical, pathological, and SRT information between the 151 patients with archived tumor tissue and the 217 patients without. Of particular importance, the cumulative incidences of BCR at 1 year ( 19 vs $26 \%$ ), 3 years ( 36 vs. $43 \%$ ), and 5 years (45 vs. $55 \%$ ) following SRT were fairly similar between these two patient groups.

The primary endpoint of this study was BCR following SRT which was defined using the same criteria as in our previous report [6], as a single post-SRT PSA value of $0.4 \mathrm{ng} / \mathrm{ml}$ or greater that exceeded the post-SRT nadir. This definition incorporates a rising profile and a threshold value (i.e., $0.4 \mathrm{ng} / \mathrm{ml}$ ) associated with clinically significant relapse [17]. The date of BCR was considered to be the date of the measured PSA value without backdating.

\section{Salvage radiation therapy information}

Patients were treated with 6-20-MV photons targeted to the prostate fossa with or without the seminal vesicles (at physician discretion) and without inclusion of the pelvic lymphatics. Treatment planning was generally performed with contrast medium in the rectum and bladder. Retrograde urethrography was also performed in many patients. Through June 1998, patients were treated with four conformal fields or a rotational field using computer tomography (CT)-based 2D-planning. After June 1998, patients were treated with ten conformal fields using CT-based 3D- 
Table 1 Comparison of clinical, pathological, and SRT information between patients with low (<115), moderate (115-130), and high ( $>130)$ COX2 staining intensity

COX-2 staining intensity

\begin{tabular}{|c|c|c|c|c|}
\hline Variable & Low $(N=49)$ & Moderate $(N=43)$ & High $(N=59)$ & $P$ value \\
\hline Pre-RP PSA (ng/mL) & $12.2(1.6,155.0)$ & $11.5(2.3,66.3)$ & $9.0(3.4,219.0)$ & 0.91 \\
\hline Pre-SRT PSA (ng/mL) & $0.6(0.1,2.6)$ & $0.6(0.1,15.3)$ & $0.5(0.1,7.9)$ & 0.24 \\
\hline SRT dose (Gy) & $64.8(60.0,70.2)$ & $66.0(60.0,70.2)$ & $64.9(58.4,72.4)$ & 0.97 \\
\hline Age & $67(53,81)$ & $67(44,77)$ & $67(50,81)$ & 0.62 \\
\hline Time from RP to SRT initiation (months) & $19.3(3.0,72.0)$ & $11.6(2.1,82.1)$ & $12.6(1.5,92.7)$ & 0.13 \\
\hline Pathological tumor stage & & & & 0.31 \\
\hline $\mathrm{T} 2$ & $18(37 \%)$ & $12(28 \%)$ & $11(19 \%)$ & \\
\hline T3a & $18(37 \%)$ & $19(44 \%)$ & $30(51 \%)$ & \\
\hline $\mathrm{T} 3 \mathrm{~b}$ & $13(27 \%)$ & $12(28 \%)$ & $18(31 \%)$ & \\
\hline Surgical margin & & & & 0.11 \\
\hline Positive & $26(53 \%)$ & $22(51 \%)$ & $41(69 \%)$ & \\
\hline Negative & $23(47 \%)$ & $21(49 \%)$ & $18(31 \%)$ & \\
\hline Gleason score & & & & 0.51 \\
\hline $3-6$ & $16(34 \%)$ & $16(37 \%)$ & $25(43 \%)$ & \\
\hline 7 & $19(40 \%)$ & $19(44 \%)$ & $23(40 \%)$ & \\
\hline $8-10$ & $12(26 \%)$ & $8(19 \%)$ & $10(17 \%)$ & \\
\hline Pre-SRT hormone therapy & & & & 0.25 \\
\hline Yes & $14(29 \%)$ & $8(19 \%)$ & $9(15 \%)$ & \\
\hline No & $35(71 \%)$ & $35(81 \%)$ & $50(85 \%)$ & \\
\hline SRT technique & & & & 0.52 \\
\hline 4 Conformal fields or a rotational field using CT-based 2D-planning & $29(59 \%)$ & $21(49 \%)$ & $35(59 \%)$ & \\
\hline 10 Conformal fields using CT-based 3D-planning & $20(41 \%)$ & $22(51 \%)$ & $24(41 \%)$ & \\
\hline Year of start of SRT & & & & 0.96 \\
\hline $1987-1992$ & $8(16 \%)$ & $8(19 \%)$ & $14(24 \%)$ & \\
\hline $1993-1998$ & $24(49 \%)$ & $18(42 \%)$ & $22(37 \%)$ & \\
\hline 1999-2003 & $17(35 \%)$ & $17(40 \%)$ & $23(39 \%)$ & \\
\hline $\mathrm{BCR}$ risk score & & & & 0.22 \\
\hline $0-2$ & $35(74 \%)$ & $32(74 \%)$ & $45(78 \%)$ & \\
\hline $3-5$ & $12(26 \%)$ & $11(26 \%)$ & $13(22 \%)$ & \\
\hline
\end{tabular}

Low, moderate, and high COX-2 staining intensity were defined as follows: low $(<115)$, moderate $(115-130)$, and high $(>130)$. The sample median (minimum, maximum) is given for numerical variables. $P$ values result from a Kruskal-Wallis rank sum test or Fisher's exact test. Information was unavailable for the following variables: pre-RP PSA $(N=5)$, Gleason score $(N=3)$, and BCR risk score $(N=3)$

$R P$ radical prostatectomy, $P S A$ prostate-specific antigen, $S R T$ salvage radiation therapy, $B C R$ biochemical recurrence, $C T$ computer tomography.

planning. A median dose of 65.0 Gy (range, 58.4-72.4 Gy) was administered in 1.8-2.0-Gy daily fractions. Intensity modulation, inverse planning, and image-guidance techniques were not used during the study time period. After SRT completion, patients were typically evaluated every 3 to 4 months for 2 years, and at 6- to 12 -month intervals thereafter.

Immunohistochemical staining

Diagnostic tissue was delivered precut onto slides. These samples were deparafinnized and rehydrated. Subsequently, antigen retrieval was performed by heating the slides in a citrate buffer in a pressure cooker for a total of $50 \mathrm{~min}$. Incubation was completed in an autostainer (Dako, Glostrup, Denmark) with an antibody directed against COX-2 (catalogue number 804-112-C050, 1:200 dilution for $1 \mathrm{~h}$, Alexis Biochemicals, Lausaen, Switzerland). A biotinylated secondary antibody (Dako LSAB 2 Kit) was overlaid for a total of $10 \mathrm{~min}$, followed by streptavidin for another $10 \mathrm{~min}$, with rinses of Tris buffer (at $\mathrm{pH} \mathrm{7 \cdot 6)}$ between staining. Diaminobenzidine chromagen was then applied for $5 \mathrm{~min}$. Slides were counterstained using haematoxylin (Dako), dehydrated, and finally coverslipped. 
Fig. 1 Cumulative incidence of biochemical recurrence (BCR) after salvage radiation therapy (SRT) for patients with low $(<115)$, moderate $(115-130)$, and high $(>130)$ COX -2 staining intensity

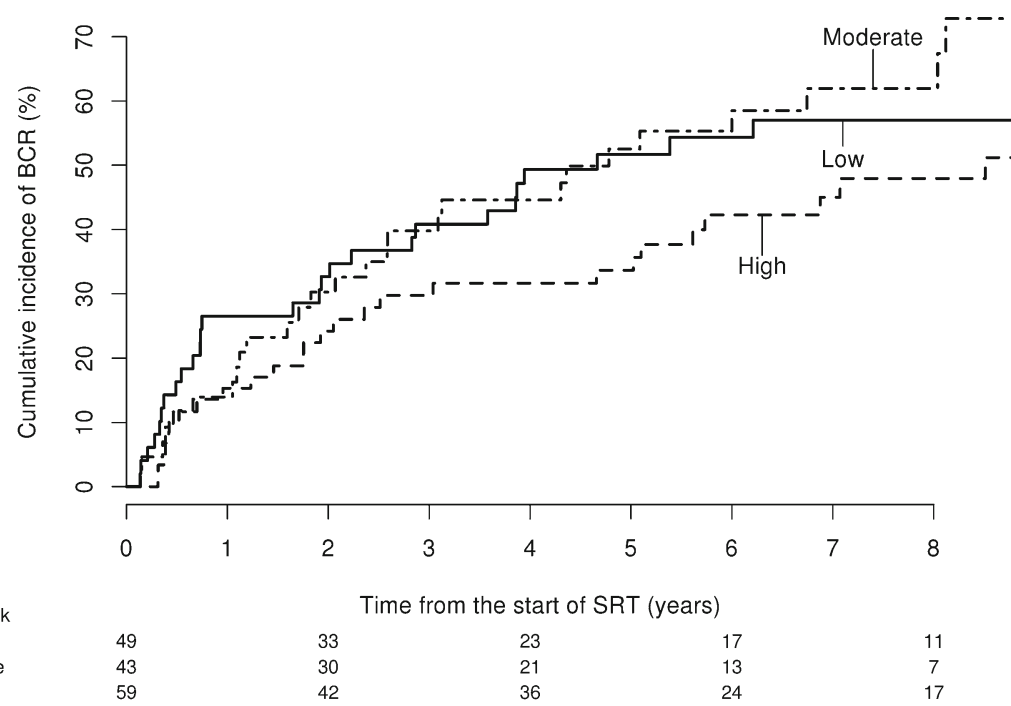

Fisher's exact test. The Kaplan-Meier method was used to estimate the cumulative incidence of BCR after the start of SRT, censoring at the date of last follow-up. Relative risks (RRs) and $95 \%$ confidence intervals (CIs) resulting from Cox proportional hazards regression models were used to evaluate the association between COX-2 staining intensity and BCR. Single variable models were utilized, as well as multivariable models adjusted for the three variables included in our BCR Risk Score [6] (pathological tumor stage, Gleason score, and pre-SRT PSA) and other patient characteristics. $P$ values $\leq 0.05$ were considered as statistically significant. Statistical analyses were performed using R Statistical Software (version 2.14.0; R Foundation for Statistical Computing, Vienna, Austria).

\section{Results}

Median COX-2 staining intensity in our cohort of 151 patients was 127 (range, 68-171). COX-2 staining intensity was low $(<115)$ in 49 patients (32\%), moderate (115-130) in 43 patients (28\%), and high (>130) in 59 patients (39\%). As detailed in Table 1, there were no significant differences in clinical, pathological, and SRT information between patients with low, moderate, and high COX-2 staining intensity (all $P \geq 0.11$ ). Median follow-up length after the start of SRT was 8.0 years (range, 0.6-20.0 years), and 87 patients (58\%) experienced BCR.

Kaplan-Meier estimated cumulative incidences of BCR after SRT are displayed in Fig. 1 and Table 2, separately for patients with low, moderate, and high COX-2 staining intensity. At 3, 5, and 8 years after SRT, cumulative incidences of BCR were 41,52 , and $57 \%$ in patients with low COX-2 staining intensity; 40,53 , and $62 \%$ in patients with moderate COX-2 staining intensity; and 30, 34, and $48 \%$ in patients with high COX-2 staining intensity. As displayed in Table 2, when comparing $\mathrm{BCR}$ between these three patient groups, there was 
no evidence of a difference in either single variable analysis $(P=0.17)$ or multivariable analysis adjusting for pre-SRT PSA, pathological tumor stage, and Gleason score $(P=0.18)$. More specifically, in multivariable analysis in comparison to patients with low COX-2 staining intensity, there was no statistically significant difference in risk of BCR for patients with moderate (RR, 1.17; $95 \%$ CI, 0.69-1.99; $P=0.69$ ) or high (RR, $0.72 ; 95 \% \mathrm{CI}, 0.42-1.22 ; P=0.18$ ) staining intensity. There was also no evidence of an association with BCR when considering COX-2 staining intensity as a continuous variable (RR, 0.83 [per 25 unit increase]; $95 \%$ CI, 0.64-1.07; $P=0.15$ ). Additional individual adjustment for other factors, such as SRT dose and year of SRT, did not alter these results (Electronic supplementary material, Table 1).

\section{Discussion}

Several investigations have demonstrated that increased COX-2 expression is predictive of poor clinical outcomes following primary treatment for prostate cancer [12-16]. Most of these studies have evaluated patient outcomes after radical prostatectomy [12-15], while the most convincing evidence has resulted from the RTOG 92-02 trial, which included patients with locally advanced prostate cancer who were treated with radiation therapy plus either short-term androgen deprivation or long-term androgen deprivation [16]. More specifically, in their analysis of 586 patients, Khor et al. demonstrated associations of increased COX-2 expression with distant metastasis, biochemical failure, and any failure. Motivated by these findings and by the need to improve the identification of men who are most likely to respond to SRT, in this study, we undertook the first evaluation to date of the ability of COX-2 staining intensity to predict BCR after SRT for a rising PSA following radical prostatectomy. In our cohort of 151 patients, we did not observe an association with BCR when considering COX-2 staining level as a three-level categorical variable based on sample tertiles or as a continuous variable in either single variable or multivariable analysis. Contrasting with the aforementioned studies utilizing patients undergoing primary prostate cancer treatment [12-16], this lack of association is likely due to our different prostate cancer population of patients with recurrent and thus uniformly more aggressive disease.

Our findings indicate that unlike clinico-pathologic features such as Gleason score, pathological tumor stage, and pre-SRT PSA, and the tumor-based biomarkers Ki-67 and B7-H3, COX-2 is not likely to be useful in selection of appropriate candidates for SRT. Though we cannot firmly conclude that no association between COX-2 staining intensity and BCR exists based on the results of our study, based on $95 \%$ confidence limits for our relative risk estimates, it is

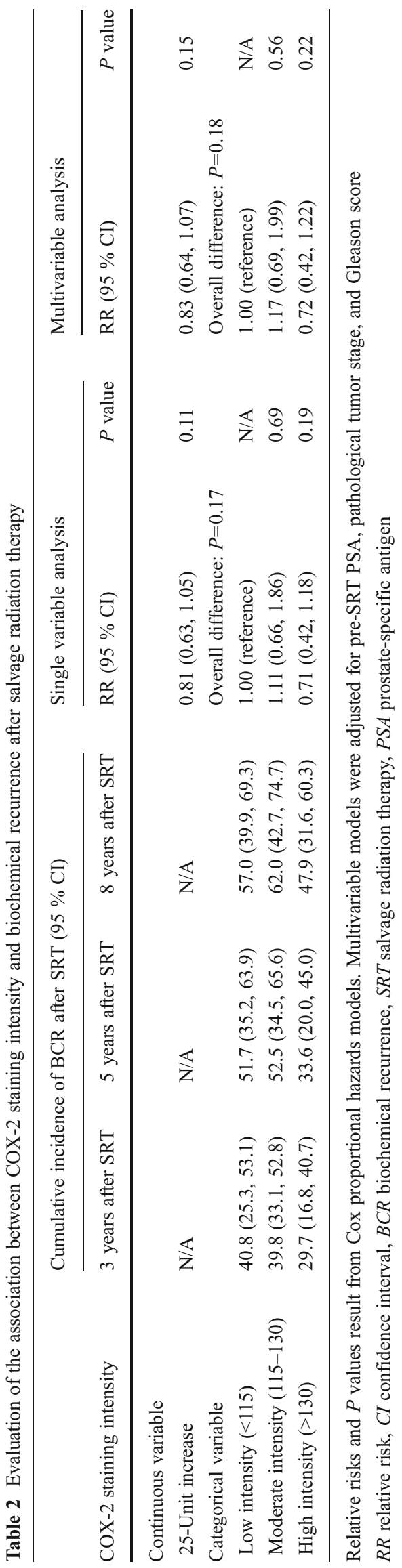


reasonable to conclude that any such association is unlikely to be strong enough to be clinically meaningful. That being said, it is important to acknowledge that power to detect an association between COX-2 staining intensity and BCR was limited, and therefore the possibility of type II error (i.e., a false-negative association) should be considered; replication in a larger cohort of SRT patients will further strengthen our findings. In addition to the relatively small sample size, other limitations of our study include the retrospective design, the primarily Caucasian population $(>98 \%)$ of our tertiary referral center, and the examination of COX-2 staining intensity using a single method (IHC analysis).

\section{Conclusion}

The results of our study, though seemingly negative owing to the lack of a statistically significant association between COX-2 staining intensity and BCR, aid in the search for biomarkers of SRT outcome. Though COX-2 clearly has an important role in prostate cancer in general, our results provide evidence that COX-2 staining is unlikely to be of use in the identification of men most likely to respond to SRT. Thus, acknowledging the need for validation in a larger patient cohort, it appears that the search for tumor-based biomarkers and other prognostic factors associated with BCR should continue elsewhere in order to further enhance patient selection for SRT.

Acknowledgment This work was supported in part from NIH-NCI CA133632-02 (PI: Pollack).

Conflict of interest statement Michael G. Heckman, Katherine S. Tzou, Alexander S. Parker, Thomas M. Pisansky, Steven E. Schild, Tracy W. Hilton, Vivek N. Patel, Liset Pelaez, Li Yan Khor, Jennifer L. Peterson, Larry C. Daugherty, Laura A. Vallow, Alan Pollack, and Steven J. Buskirk declare that they have no conflict of interest.

Human or animal subjects This article does not contain any studies with human or animal subjects performed by any of the authors.

\section{References}

1. Patel AR, Stephenson AJ (2011) Radiation therapy for prostate cancer after prostatectomy: adjuvant or salvage? Nat Rev Urol 8:385-392

2. Pound CR, Partin AW, Eisenberger MA, Chan DW, Pearson JD, Walsh PC (1999) Natural history of progression after PSA elevation following radical prostatectomy. JAMA 281:1591-1597

3. Trock BJ, Han M, Freedland SJ, Humphreys EB, DeWeese TL, Partin AW, Walsh PC (2008) Prostate cancer-specific survival following salvage radiotherapy vs observation in men with biochemical recurrence after radical prostatectomy. JAMA 299:2760-2769

4. Freedland SJ, Humphreys EB, Mangold LA, Eisenberger M, Dorey FJ, Walsh PC, Partin AW (2005) Risk of prostate cancer- specific mortality following biochemical recurrence after radical prostatectomy. JAMA 294:433-439

5. Choo R (2010) Salvage radiotherapy for patients with PSA relapse following radical prostatectomy: issues and challenges. Cancer Res Treat 42:1-11

6. Buskirk SJ, Pisanksy TM, Schild SE, Macdonald OK, Wehle MJ, Kozelsky TF, Collie AC, Ferrigni RG, Myers RP, Prussak KA, Heckman MG, Crook JE, Parker AS, Igel TC (2006) Salvage radiotherapy for isolated prostate specific antigen increase after radical prostatectomy: evaluation of prognostic factors and creation of a prognostic scoring system. J Urol 176:985-990

7. Stephenson AJ, Scardino PT, Kattan MW, Pisansky TM, Slawin KM, Klein EA, Anscher MS, Michalski JM, Sandler HM, Lin DW, Forman JD, Zelefsky MJ, Kestin LL, Roehrborn CG, Catton CN, DeWeese TL, Liauw SL, Valicenti RK, Kuban DA, Pollack A (2007) Predicting the outcome of salvage radiation therapy for recurrent prostate cancer after radical prostatectomy. J Clin Oncol 25:2035-2041

8. Parker AS, Heckman MG, Wu KJ, Crook JE, Hilton TW, Pisansky TM, Bernard JR, Schild SE, Khor LY, Hammond EH, Pollack A, Buskirk SJ (2009) Evaluation of ki-67 staining levels as an independent biomarker of biochemical recurrence after salvage radiation therapy for prostate cancer. Int J Radiat Oncol Biol Phys 75:1364-70

9. Parker AS, Heckman MG, Sheinin Y, Wu KJ, Hilton TW, Diehl NN, Pisansky TM, Schild SE, Kwon ED, Buskirk SJ (2011) Evaluation of B7-H3 expression as a biomarker of biochemical recurrence after salvage radiation therapy for recurrent prostate cancer. Int J Radiat Oncol Biol Phys 79:1343-1349

10. Heckman MG, Parker AS, Wu KJ, Hilton TW, Ko SJ, Pisansky TM, Schild SE, Khor LY, Hammond EH, Pollack A, Buskirk SJ (2012) Evaluation of MDM2, p16, and p53 staining levels as biomarkers of biochemical recurrence following salvage radiation therapy for recurrent prostate cancer. Prostate 72:1757-1766

11. Hussain T, Gupta S, Mukhtar H (2003) Cyclooxygenase-2 and prostate carcinogenesis. Cancer Lett 191:125-135

12. Rubio J, Ramos D, López-Guerrero JA, Iborra I, Collado A, Solsona E, Almenar S, Llombart-Bosch A (2005) Immunohistochemical expression of Ki-67 antigen, cox-2 and Bax/Bcl-2 in prostate cancer: prognostic value in biopsies and radical prostatectomy specimens. Eur Urol 48:745-751

13. Di Lorenzo G, De Placido S, Autorino R, De Laurentiis M, Mignogna C, D'Armiento M, Tortora G, De Rosa G, D'Armiento M, De Sio M, Bianco AR, D'Armiento FP (2005) Expression of biomarkers modulating prostate cancer progression: implications in the treatment of disease. Prostate Cancer Prostatic Dis 8:54-59

14. Cohen BL, Gomez P, Omori Y, Duncan RC, Civantos F, Soloway MS, Lokeshwar VB, Lokeshwar BL (2006) Cyclooxygenase-2 $(\mathrm{COX}-2)$ expression is an independent predictor of prostate cancer recurrence. Int J Cancer 119:1082-1087

15. Di JM, Zhou J, Zhou XL, Gao X, Shao CQ, Pang J, Sun QP, Zhang Y, Ruan XX (2009) Cyclooxygenase-2 expression is associated with vascular endothelial growth factor-C and lymph node metastates in human prostate cancer. Arch Med Res 40:268-275

16. Khor LY, Bae K, Pollack A, Hammond ME, Grignon DJ, Venkatesan VM, Rosenthal SA, Ritter MA, Sandler HM, Hanks GE, Shipley WU, Dicker AP (2007) COX-2 expression predicts prostate-cancer outcome: analysis of data from the RTOG 92-02 trial. Lancet Oncol 8:912-920

17. Stephenson AJ, Kattan MW, Eastham JA, Dotan ZA, Bianco FJ Jr, Lilja H, Scardino PT (2006) Defining biochemical recurrence of prostate cancer after radical prostatectomy: a proposal for a standardized definition. J Clin Oncol 24:3973-3978 